\title{
Geodesic oscillations and the weakly coherent mode in the I-mode of ASDEX Upgrade
}

\author{
P. Manz ${ }^{1,2}$, P. Lauber ${ }^{2}$, V.E. Nikolaeva ${ }^{3,1,2}$, T. Happel ${ }^{2}$, F. Ryter ${ }^{2}$, G. Birkenmeier ${ }^{1,2}$, A. Bogomolov ${ }^{4}$, G.D. \\ Conway $^{2}$, M.E. Manso ${ }^{3}$, M. Maraschek ${ }^{2}$, D. Prisiazhniuk ${ }^{1,2}$, E. Viezzer ${ }^{2}$ and the ASDEX Upgrade Team \\ 1 Physik-Department E28, Technische Universität München, \\ James-Franck-Str. 1, 85748 Garching, Germany \\ 2 Max-Planck-Institut für Plasmaphysik, Boltzmannstr. 2, 85748 Garching, Germany \\ 3 Instituto de Plasmas e Fusão Nuclear, Instituto Superior Técnico, \\ Universidade Técnica de Lisboa, 1049-001 Lisboa, Portugal \\ ${ }^{4}$ FOM-Insitute DIFFER, Dutch Institute for Fundamental Energy Research, 3430 BE Nieuwegein, The Nederlands
}

(Dated: May 6, 2015)

\begin{abstract}
Density fluctuations in I-mode discharges in ASDEX Upgrade are studied. The I-mode specific weakly coherent mode (WCM) appears at the transition from L to I-mode. The WCM but also the turbulence in general are strongly modulated by a low frequency mode which can be related to the geodesic acoustic mode (GAM). The GAM induces an energy transfer away from the central WCM frequency, indicating an underlying instability responsible for the WCM. During the I-mode magnetic fluctuations close to the WCM frequency are intensified, which can be assigned to the geodesic Alfvénic oscillation. The geodesic Alfvénic oscillation is present already in L-mode, does not follow changes of frequency of the WCM, therefore it is not responsible for the WCM.
\end{abstract}

PACS numbers:

\section{INTRODUCTION}

The I-mode [1] typical appears in the unfavorable configuration where the ion $\nabla B$ drift is directed away from the $\mathrm{X}$-point. In the unfavorable configuration the $\mathrm{H}$ mode power threshold is about 2 times higher compared to the favorable configuration. The I-mode is an improved energy confinement regime exhibiting an edge energy transport barrier but without an accompanying particle transport barrier. This has several benefits [2]:

(i) H-mode like energy confinement

(ii) weak degradation of energy confinement with increasing heating power

(iii) less accumulation of helium ash due to the lack of the particle transport barrier

(iv) absence of ELMs due to the smaller edge pressure gradient compared to $\mathrm{H}$-mode

The understanding of the decoupling of heat and particle transport in the I-mode is also valuable for the understanding of the underlying mechanisms controlling transitions to high-energy confinement regimes in general.

Previous works showed that at the transition to the I-mode low frequency broadband fluctuations decrease while simultaneously a weakly coherent mode (WCM) in the density and magnetic fluctuations at high frequencies $(f \sim 100-300 \mathrm{kHz})$ develops [3]. The WCM appears in the electron temperature $T_{e}$ pedestal region $(0.95<$ $r / a<1.0$ in Alcator C-Mod) [2] with a tail towards the pedestal top [4]. Fluctuation levels of the WCM in the density, magnetics and temperature are around $\tilde{n} / n \sim 10-16 \%, \tilde{B} / B \sim 0.01-0.02 \%$ and $\tilde{T}_{e} / T_{e} \sim 1-2$ $\%$, respectively [3-5]. The WCM is thought to be responsible for the regulation of the particle transport and therefore the cause of the L-mode like particle transport in the I-mode. While the WCM appears quite broadband in frequency space it is rather narrow in wavenumber space and has short wavelengths $\left(k_{\theta}=1.3 \pm 0.5 \mathrm{~cm}^{-1}\right)$ [4]. The WCM propagates in the electron diamagnetic direction in both the laboratory [2] and $E \times B$ frame [4]. In the $E \times B$ frame the propagation velocity is roughly a factor three below the electron diamagnetic velocity [4]. The WCM can manifest at low $q_{95}$ and low collisionality and promptly disappears at the transition to the H-mode $[2]$.

The nonlinear analysis in Ref. [4] shows a nonlinear coupling of the geodesic acoustic mode (GAM) and the WCM and also an energy transfer from the WCM into the GAM which constitutes a saturation mechanism for the WCM. Another effect is that the GAM scatters the energy of the central WCM peak by transferring energy below the WCM frequency from higher to lower and above the WCM frequency from lower to higher frequencies. Thereby the GAM is responsible for the broadband feature of the WCM. Indeed such a coupling to a rather lowwavenumber mode as the GAM is necessary to preserve the narrow width in wavenumber of the WCM. Therefore the central frequency peak of WCM is acting as a source of the WCM. A basic conclusion from this is that there must by an underlying instability responsible for the WCM.

Up to now studies of turbulence in I-mode are restricted to Alcator C-Mod. Investigations in different experiments as the present one in ASDEX Upgrade are helpful to elaborate similarities and differences of turbulence in I-mode between Alcator C-Mod and ASDEX Upgrade and which phenomena are I-mode specific in general. 


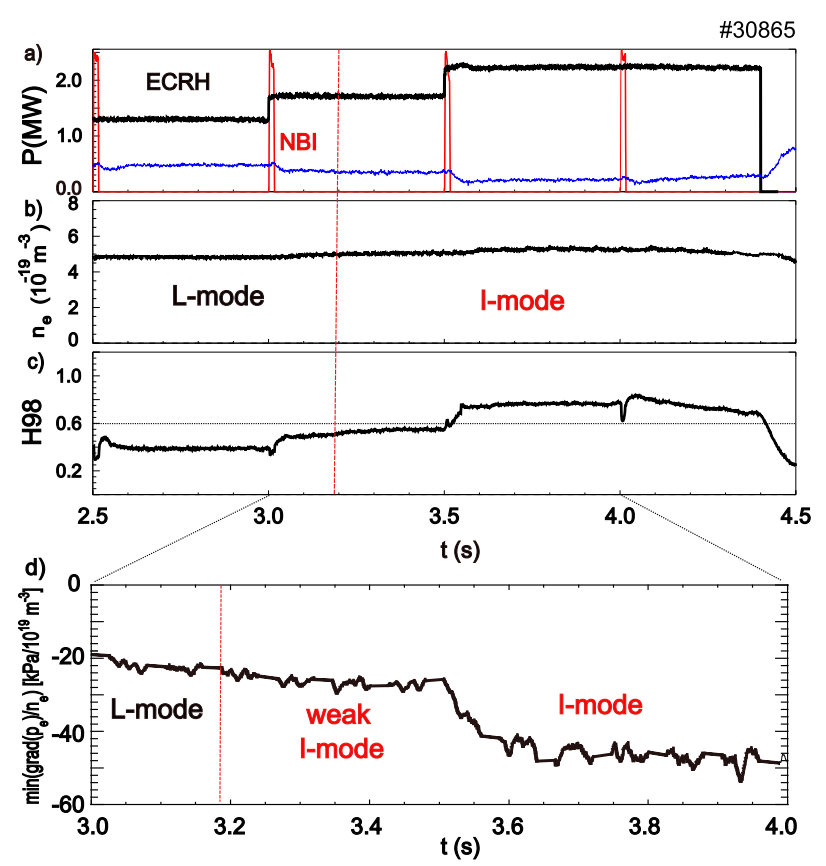

FIG. 1: a) Heating power b) line averaged density c) H98 confinement parameter and $\mathrm{d}) \min \left(\left(\nabla p_{e}\right) / \mathrm{n}\right)$. The vertical line indicates the L-I transition.
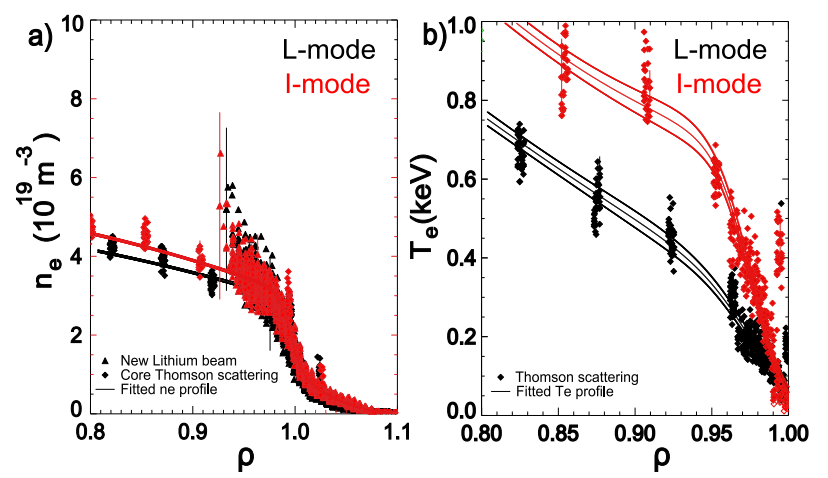

FIG. 2: a) Electron density and b) electron temperature profiles at the plasma edge for the L-mode (black at $2.5 \mathrm{~s}$ ) and I-mode (red at $4.0 \mathrm{~s}$ ) case. The development of a temperature pedestal is clearly observable, whereas the density is only subject to marginal changes. A direct comparison of the profiles is not allowed as the heating power is different.

\section{WEAKLY COHERENT MODE}

Experiments were carried out on the ASDEX Upgrade tokamak, which has major and minor horizontal radii of $R_{0}=1.65 \mathrm{~m}$ and $a=0.5 \mathrm{~m}$, respectively. The toroidal magnetic field strength was $B_{t}=-2.5 \mathrm{~T}$ and the plasma current was $I_{p}=1 \mathrm{MA}$. In the upper-single null configuration the ion $\nabla B$ drift is directed away from the $\mathrm{X}$-point. The basic discharge setup is shown in Fig. 1. The heating power of the ECRH has been increased every $500 \mathrm{~ms}$ (Fig. 1a). With increasing heating power, the density

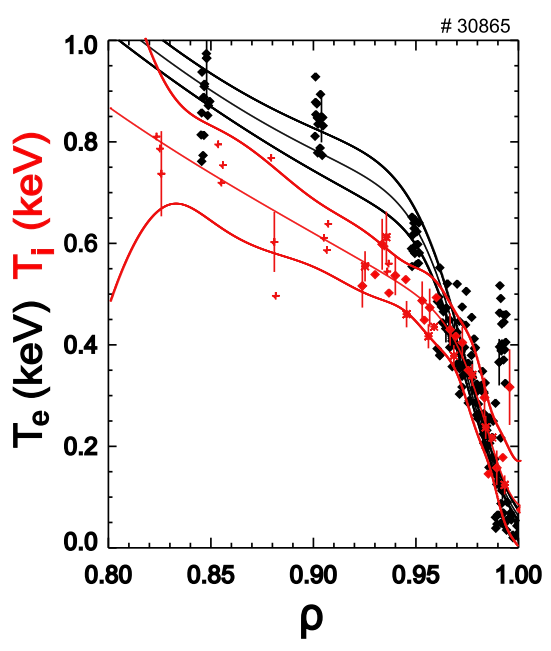

FIG. 3: Electron (black) and ion (red) temperature profiles at the plasma edge for the I-mode (at $4.0 \mathrm{~s}$ ). Electron temperature has been measured via Thomson scattering, ion temperature via charge exchange spectroscopy.

stays rather constant (Fig. 1b) and the confinement increases with respect to the H98 H-mode scaling (Fig. 1c). In this case, the transition from L to I mode cannot be identified with accuracy because clear signatures are missing. However, at $t=3.18 \pm 0.05$ s electron temperature and pressure gradients $\nabla p_{e}$ slightly steepen up as it can be seen by a small continuous drop in $\min \left(\nabla p_{e} / n\right)$ starting at $3.18 s$ (Fig. 1d) and the confinement increases slightly at constant heating power. This spontaneous confinement transition is considered as a transition to a weak I-mode. At this density, $T_{e}$ and $T_{i}$ are close to each other at the plasma edge $(\rho>0.95)$ as shown in Fig. 3 and one may assume $\left(\nabla p_{e}\right) / n \sim\left(\nabla p_{i}\right) / n e$ which corresponds to the simple neoclassical estimate of the radial electric field [6]. One may speculate that the confinement transition is related to changes in the radial electric field or its contributions [4]. With additional heating power at $t=3.5 \mathrm{~s}, H 98$ increases above 0.6 and a clear I-mode develops. The ion (not shown here) and electron temperature gradients strongly increase, while the density gradient only increases slightly (Fig. 2).

The density fluctuations shown in Figs. 4 and 5a are measured by hopping reflectometry diagnostic. Hopping reflectometer is dedicated to density fluctuation measurements. It operates in O-mode using two channels: Qband (33-49.2 GHz) and V-band (49.4-72 GHz) featuring individual single antennae located at the LFS, approximately at the tokamak midplane. The diagnostic is equipped with heterodyne detection enabling to extract phase directly. Density fluctuations in Figs. 4 and 5 a were obtained for the probing frequency of $45 \mathrm{GHz}$. The cut-off density $n_{e}=2.5 \cdot 10^{19} \mathrm{~m}^{-3}$ is located at $\rho=0.98 \pm 0.01$. The electron density fluctuation level of the WCM estimated using the 1D C. Fanack model [7] was found to be between 6 and $13 \%$. After $t=3.5 \mathrm{~s}$ 
we observe two bands in frequency space, one at low frequencies $(f<30 \mathrm{kHz})$ and one at higher frequencies $(80<f<150 \mathrm{kHz})$. Although the mode is not as pronounced here, this behavior is very similar to the WCM observed in Alcator C-Mod [3, 4]. Already around $t=3.2 \mathrm{~s}$ where the pedestal forms a slight divergence in frequency space can be adumbrated. These modes can also be observed at the probing frequency of $48 \mathrm{GHz}$ $\left(2.84 \cdot 10^{19} \mathrm{~m}^{3}\right)$, as well as at 38 and $39 \mathrm{GHz}\left(1.78 \cdot 10^{19}\right.$ $\mathrm{m}^{3}$ and $1.87 \cdot 10^{19} \mathrm{~m}^{3}$, respectively), but not so clearly pronounced. Then, considering this density range from 1.8 to $2.86 \cdot 10^{19} \mathrm{~m}^{3}$, we can conclude that on the low field side (LFS) this mode is located at $\rho=0.97-0.99 \pm 0.01$ corresponding to the minimum $E_{r}$ in I-modes at ASDEX Upgrade [8] and consistent with the recent observation on Alcator C-Mod [4]. The minimum of $\nabla\left(p_{e}\right) / n e$ is located at $\rho=0.99$. At all frequencies of the hopping reflectometer below $38 \mathrm{GHz}(33,36$ and $37 \mathrm{GHz})$ and all frequencies higher $48 \mathrm{GHz}$, which were for $\mathrm{V}$ band between 51.5 and $64 \mathrm{GHz}$, these coherent modes were not seen. The coherent modes are also observed on the high field side (HFS) by the frequency modulated continuous wave (FMCW) reflectometer probing $n_{e}=1.6 \cdot 10^{19} \mathrm{~m}^{-3}$ (probing frequency $36 \mathrm{GHz}$ ). The location of those modes was not yet determined because in this discharge, the FMCW reflectometer was set up in fixed frequency mode for density fluctuation studies, therefore no simultaneous HFS/LFS density profile measurements were done. Profiles measured at the LFS by interferometer, lithium beam and Thomson scattering, could be mapped on the HFS but this is not straightforward, because previous results of reflectometry profiles observed in other I mode discharges seems to indicate that there might be an asymmetry of density profiles between LFS and HFS. The mode is not observed in K-band (probing the SOL region) and $\mathrm{V}$ band channels.

\section{MODULATION BY GEODESIC ACOUSTIC MODE}

It can be expected that the WCM is modulated by a GAM as in Alcator C-Mod [4]. GAMs can be directly observed in velocity fluctuations measured for example by Doppler reflectometry. Furthermore GAMs show up in bispectra as they are nonlinearly driven and modulate the turbulence at the GAM frequency $[9,10]$.

The intrinsic modulation of all higher frequencies by the GAM can be used for the detection of GAMs [11]. The modulation shows up in the envelope of the fluctuations. The envelope of the density fluctuations is given by $\operatorname{Env}(t)=\sqrt{\operatorname{Re}(\tilde{n}(t))^{2}+\operatorname{Im}(\tilde{n}(t))^{2}}$. We measure the real part directly, the imaginary part can be deduced in frequency space from the Fourier transform $\tilde{n}(\omega)$ and is given by $-i \cdot \operatorname{sgn}(\omega) \tilde{n}(\omega)$. The inverse Fourier transform of this relation is the convolution $n(t) * 1 /(\pi t)=\mathcal{H}(n(t))$ which is the Hilbert transform $\mathcal{H}$ by definition. Therefore the envelope is given by $\operatorname{Env}(t)=\sqrt{(\tilde{n}(t))^{2}+(\mathcal{H}(\tilde{n}(t)))^{2}}$.

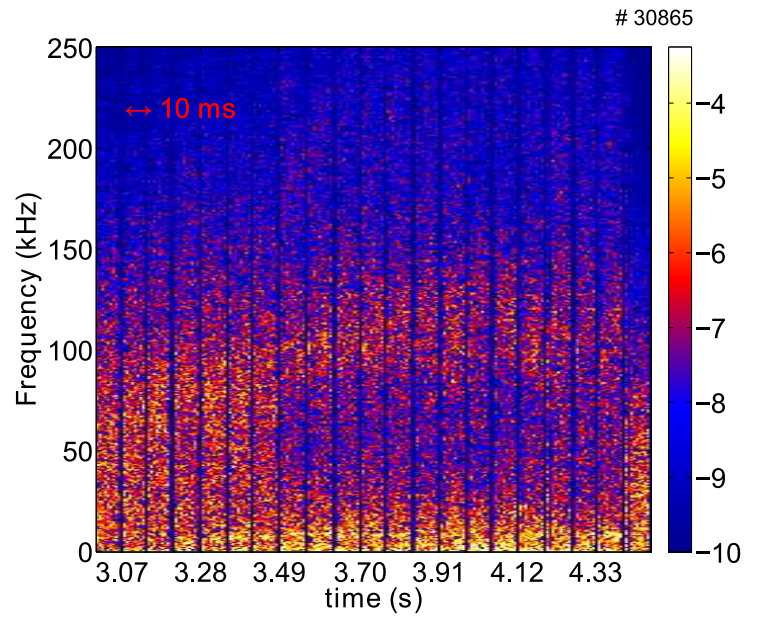

FIG. 4: Spectrogram of density fluctuations from hopping reflectometry. Amplitudes are shown in logarithmic representation. Note that the plot is not continuous. Subwindows of $10 \mathrm{~ms}$ length corresponding only to the cut-off layer density of $n_{e}=2.5 \cdot 10^{19} \mathrm{~m}^{-3}$ are shown. The hopping reflectometry measures every $70 \mathrm{~ms}$ at this cut-off layer for $10 \mathrm{~ms}$.

As reference for L-mode (I-mode), data from about 2.5 $\mathrm{s}$ to $2.75 \mathrm{~s}(3.75 \mathrm{~s}$ to $4.0 \mathrm{~s})$ is compared in Fig. $5 \mathrm{~b}$. The envelope of density fluctuations measured by reflectometry has been estimated from fluctuations above $400 \mathrm{kHz}$. For better comparison with the density fluctuations the envelope in Fig. 5b is shown for a subwindow size of $1 \mathrm{~ms}$ corresponding to a frequency resolution of $1 \mathrm{kHz}$. Figures $5 \mathrm{a}$,b show the spectra of density fluctuations as well as that of the envelope of fluctuations high pass filtered above $400 \mathrm{kHz}$. In I-mode a rather broadband mode around $130 \mathrm{kHz}$ is observed (the WCM). Turbulence is slightly reduced in the frequency range from 20 to 80 $\mathrm{kHz}$ and increased at higher frequencies (above $80 \mathrm{kHz}$ ). The transfer of free energy from low to high frequencies is usual for the $E \times B$ nonlinearity, which results in a direct cascade $[12,13]$. However, by means of charge exchange spectroscopy the mean poloidal velocity has been measured during beam blips which is used to translate the obtained frequency spectra into the plasma frame. The wavenumber of the WCM found at $k_{\theta}=2 \pi f / v_{\theta} \approx 1.5$ $\mathrm{cm}^{-1}$ is similar to the Alcator C-Mod results [4]. However, it should be noted that the Doppler data is taken at $k_{\perp} \sim 10 \mathrm{~cm}^{-1}$ and the WCM is not that narrow in wavenumber space. As seen in Fig. 5d the turbulence level in the I-mode is reduced and the increase in fluctuation level at high frequency Fig. 5a is due to the increased Doppler shift due to the increased rotation in the I-mode and not due to an increase of energy transfer from low to high frequencies by the GAM. The envelope at low frequencies around $8 \mathrm{kHz}$ is about 2 orders of magnitude larger in I-mode pointing to a strong modulation of the turbulence at the GAM frequency. Compared to $c_{s} / R_{0}$ this frequency seems rather low, however as we will see 
a)

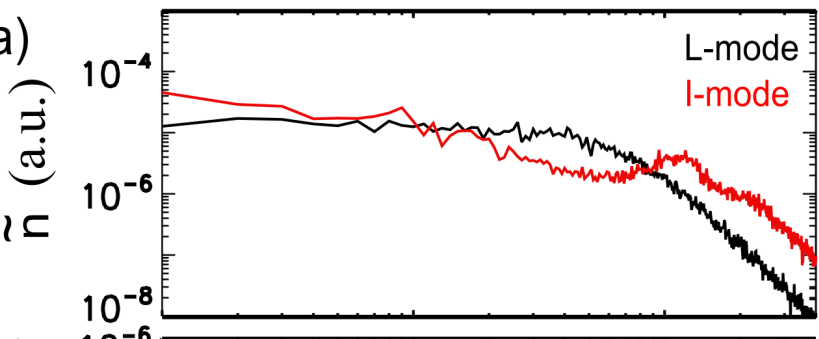

b)
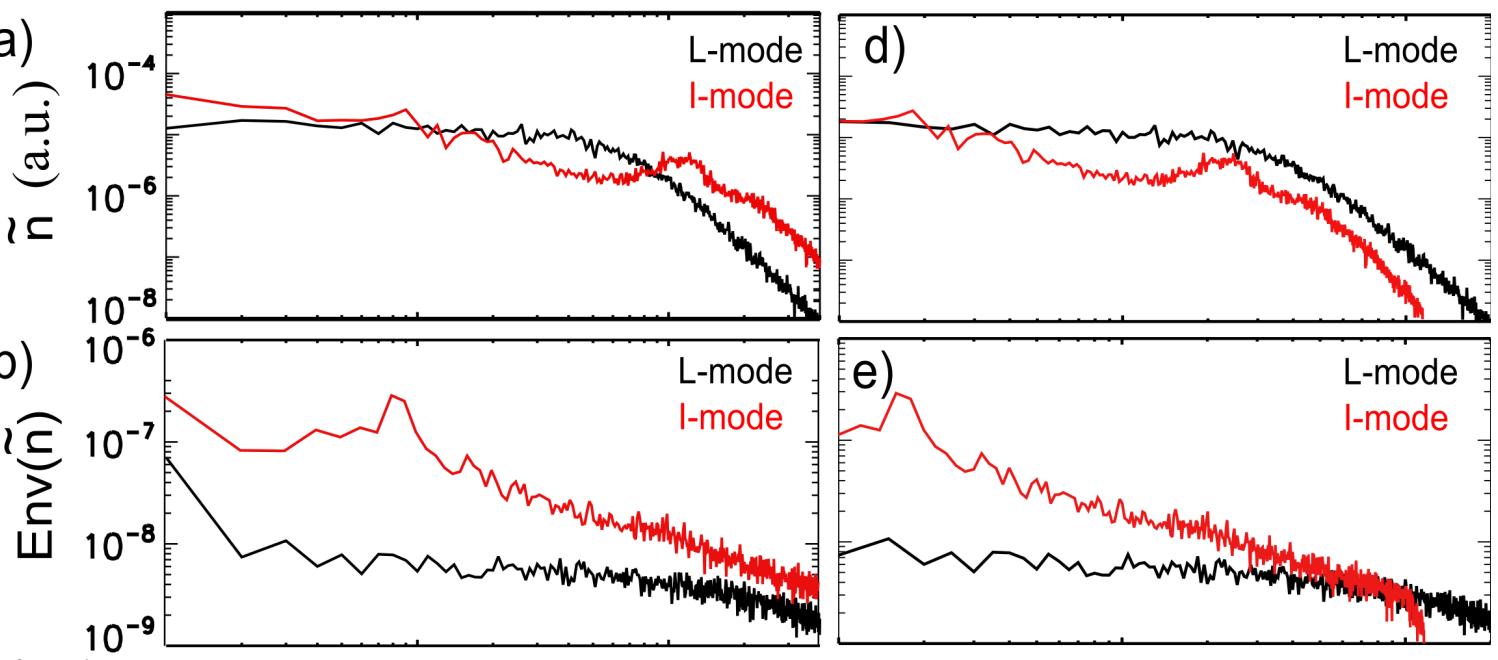

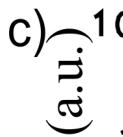

l. $\omega^{\circ}$

1.

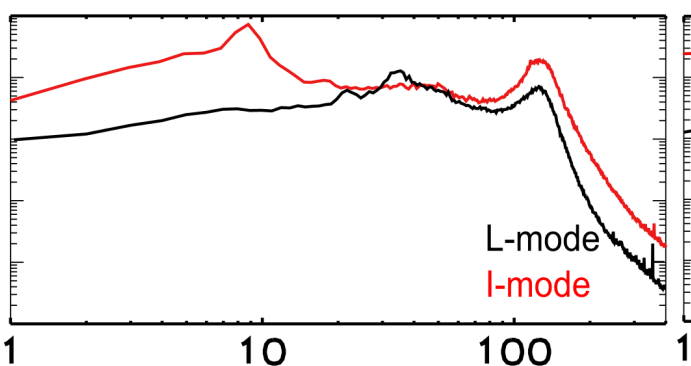

$\mathrm{f}(\mathrm{kHz})$

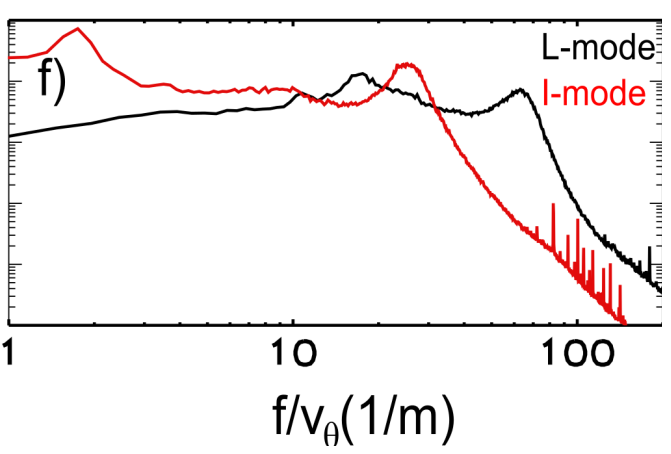

FIG. 5: (a) Spectrum of density fluctuations from hopping reflectometry, (b) its envelope deduced from density fluctuations and (c) magnetic fluctuations $\dot{B_{\theta}}$ below $400 \mathrm{kHz}$ in L and I-mode. Spectra are also shown in the plasma frame $(\mathrm{d}-\mathrm{f})$ with $v_{\theta} \approx-2$ $\mathrm{km} / \mathrm{s}$ in L-mode and $v_{\theta} \approx-5 \mathrm{~km} / \mathrm{s}$ in I-mode, respectively. Negative velocities are in the electron diamagnetic direction.

in the following section this frequency agrees well with the theoretical prediction of the GAM frequency.

A study of the free energy transfer as done in Ref. [4] would require to estimate the $E \times B$ nonlinearity $\operatorname{Re}\left(\tilde{n}^{*}(f)\left(\left\langle\hat{b} \times \nabla_{\perp} \tilde{\phi}\left(f_{2}\right) \cdot \nabla_{\perp}\right) \tilde{n}\left(f_{1}\right)\right\rangle\right)$ with magnetic field direction $\hat{b}$ and therefore the cross-bicoherence between density and potential fluctuations. Density fluctuations $\tilde{n}$ can be deduced from reflectometry. Potential fluctuation measurements are not available at ASDEX Upgrade. But with Doppler reflectometry poloidal velocities can be measured. Velocity fluctuations are approximated by the center of gravity of the Doppler shift given by

$$
c_{g r}=\frac{\int d f f S(f)}{\int d f S(f)}
$$

with the power spectrum $S(f)$ of the Doppler reflectometer heterodyne signal. The center of gravity is calculated for subwindows of $1 \mu \mathrm{s}$ length corresponding to 25 points (25 MHz acquisition). The amplitude of the Doppler reflectometer heterodyne signal is proportional to density fluctuations $\tilde{n}$. Also the amplitude of the reflectometer heterodyne signal is averaged over $1 \mu \mathrm{s}$. It should be pointed out that the center of gravity is measured in absolute units $(\mathrm{Hz})$ which in general are not equal to the Doppler shift, so that no statement on the absolute value of the velocity fluctuations can be made.

To investigate the nonlinear coupling between WCM and GAM, the cross-bicoherence can be estimated from the density fluctuations $\tilde{n}$ and the center of gravity $\tilde{c}_{g r}$

$$
\hat{b}\left(f_{1}, f_{2}\right)=\sqrt{\frac{\left\|\left\langle\tilde{n}\left(f_{1}\right) \tilde{c}_{g r}\left(f_{2}\right) \tilde{n}^{*}\left(f_{1}+f_{2}\right)\right\rangle\right\|^{2}}{\left\langle\left\|\tilde{n}\left(f_{1}\right) \tilde{c}_{g r}\left(f_{2}\right)\right\|\right\rangle^{2}\left\langle\left\|\tilde{n}\left(f_{1}+f_{2}\right)\right\|\right\rangle^{2}}} .
$$

This cross-bicoherence gives the degree of phase locking between the three different modes $\tilde{n}\left(f_{1}\right), \tilde{c}_{g r}\left(f_{2}\right)$ and $\tilde{n}\left(f_{1}+f_{2}\right)$ and takes values between zero and one. Phase locking is a necessary condition for nonlinear coupling. A coupling of the GAM with the WCM as expected from recent Alcator C-Mod experiments [4] should be observed in the cross-bicoherence if such coupling exists. The bispectral analysis is done for a subwindow size of $500 \mu \mathrm{s}$, resulting in 90 ensembles and a frequency resolution of 2 $\mathrm{kHz}$. The corresponding significance level is 0.011 .

L-mode (I-mode) data is taken from $2.4 \mathrm{~s}$ to $2.8 \mathrm{~s}(3.7$ $\mathrm{s}$ to $4.1 \mathrm{~s}$ ) and the bispectra are shown in Figs. 6a and 
$6 \mathrm{~b}$, respectively. In the L-mode no pronounced modes can be found. In I-mode a coupling of the center of gravity proportional to velocity fluctuations at low frequency $(\sim 10 \mathrm{kHz})$ with the broadband turbulence $(>200 \mathrm{kHz})$ is observed. A pronounced coupling of these fluctuations at $10 \mathrm{kHz}$ with the WCM $(70-140 \mathrm{kHz})$ is found. The cross-bicoherence at 0.14 strongly exceeds the significance level. The WCM itself also is nonlinear coupled to other frequencies. The velocity fluctuations of the WCM are coupled to low frequency density fluctuations $\left(f_{1}<20 \mathrm{kHz}\right)$ and to fluctuations near the WCM frequency. Additionally coupling of velocity fluctuations at the WCM frequencies with higher frequencies in the density fluctuations are observed $\left(f_{1}>200 \mathrm{kHz}\right.$ for $f_{2}=+f_{W C M}$ and $f_{1}>300 \mathrm{kHz}$ for $\left.f_{2}=-f_{W C M}\right)$. This might indicate that the bursts observed in the Doppler reflectometry [14] in the density fluctuations are nonlinearly generated by the WCM.

Also not shown here, the bispectra inferred from the envelope analysis from the FMCW reflectometer show the nonlinear coupling of the GAM with the WCM. Compared against each other the bispectra deduced from the Doppler reflectometer show more details. However, if no Doppler reflectometer is available the method proposed by Nagashima et al. [11] can be applied to conventional reflectometer data and can turn out useful for the analysis of quasi-coherent modes in general. Quasi-coherent modes show a high degree of phase locking, which is studied by bispectral analysis and a necessary condition for nonlinear coupling [13].

\section{GEODESIC ALFVÉNIC MODE}

Very similar features as in the density fluctuations can be also observed in the magnetics as shown by Figs. $5 \mathrm{c}$ and 7. Also the low frequency fluctuations at $8 \mathrm{kHz}$ are observed once the I-mode is accessed from $t=3.2 \mathrm{~s}$ on (Fig. 7). As a high frequency zonal flow the GAM could potentially lead to the spontaneous confinement transition at $t=3.2 \mathrm{~s}$ and the drop in the diamagnetic contribution of the radial electric field $\left(\nabla p_{i}\right) / n e$. This has also been speculated in Ref. [4]. Note that magnetic fluctuations at the frequency of the WCM $(130 \mathrm{kHz})$ can be detected well before the L-I transition. This is in contrast to the observations in Alcator C-Mod, where the frequency of the magnetic fluctuations follow the frequency of the WCM which increases during the beginning of the I-mode [2]. Therefore the magnetic fluctuations investigated in detail in the following are probably not related to the WCM even though they appear at a similar frequency.

The GAM is the oscillation between the zonal flow and its pressure sideband. These pressure sidebands are not only coupled to the turbulence but also to the global Alfvénic oscillation [15]. If the high frequency magnetic oscillations at the WCM frequency are related to Alfvénic modes they should scale with $\sim 1 / \sqrt{n}$. This scaling can be investigated in a discharge with a steady I-mode a) L-mode

\# 30865

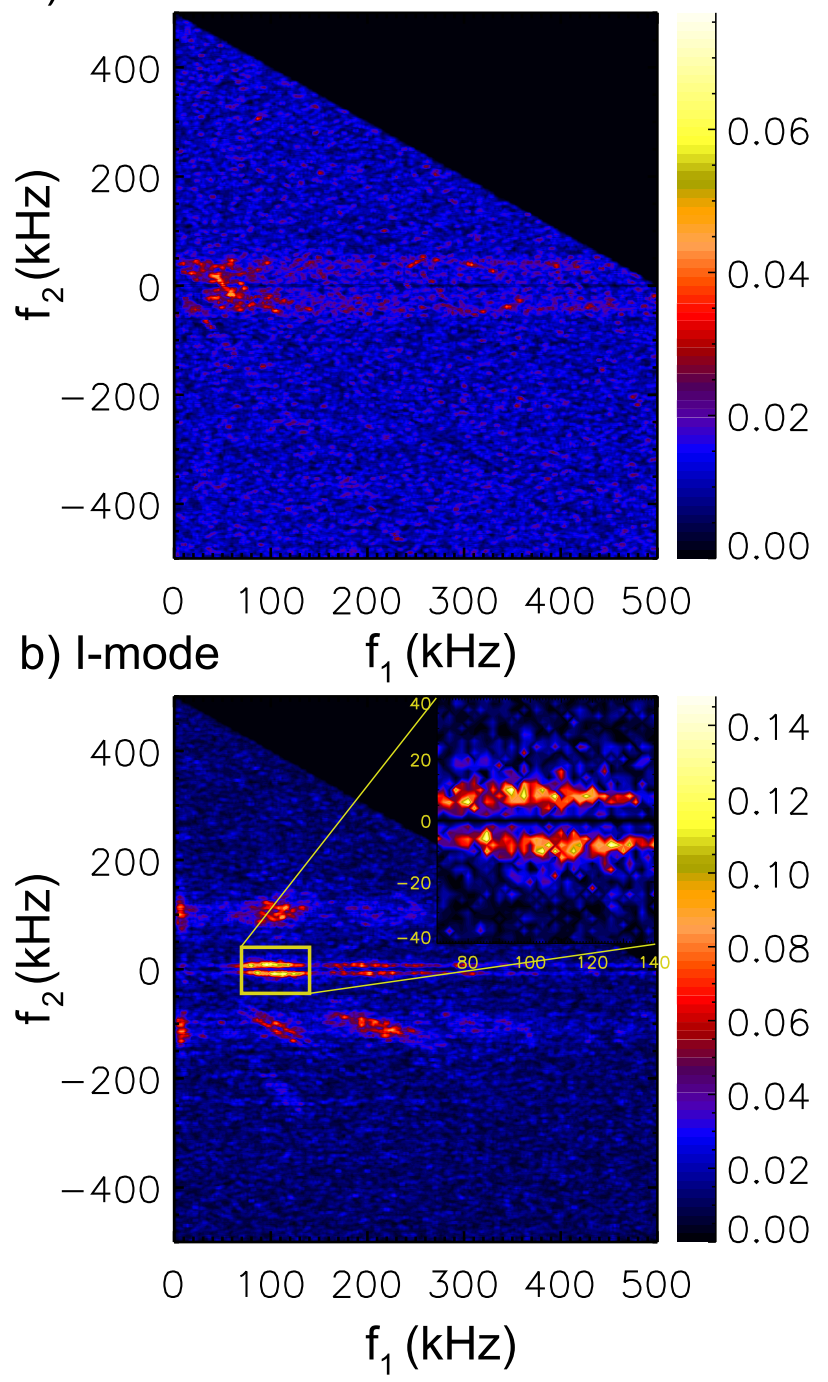

FIG. 6: Bispectrum of density fluctuations from Doppler reflectometry in (a) L-mode and (b) I-mode. The frequency of density fluctuations $f_{1}$ couples with frequencies in velocity fluctuations $f_{2}$ to density fluctuations $f=f_{1}+f_{2}$. Data is taken at $80 \mathrm{GHz}$.

phase where the density is varying smoothly over time. Figure 8 shows the frequency of the maximum power in the range between 100 and $200 \mathrm{kHz}$ and therefore tracks the frequency changes of the high frequency magnetic fluctuations which appear also around $140 \mathrm{kHz}$ in this discharge and $\sim 1 / \sqrt{n}$ from the edge density measured with interferometry. To compare the time evolution both are normalized to their values at the beginning of the Imode. Of course this comparison is just a plausibility check as both signals are not matched in physical space. In the long time behavior the high frequency oscillations in the magnetic field follow the trend of $\sim 1 / \sqrt{n}$ and both increase by about $5 \%$ which points to an Alfvénic mode. The two signals do not exhibit a clear correlation 


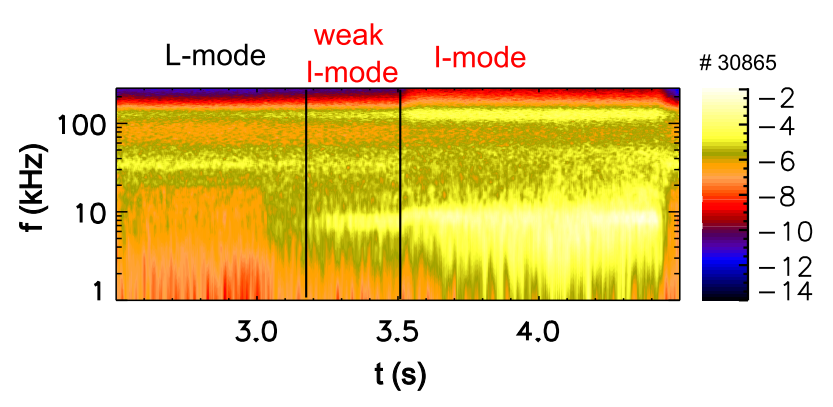

FIG. 7: Spectogram of fluctuations in $\dot{B}_{\theta}($ C09-09) in logarithmic amplitude representation.

on shorter time scales, or in shorter I-mode phases.

Alfvénic eigenmodes with $n \neq 0$ are called beta in- duced Alfvénic eigenmodes, those with $n=0$ are called geodesic Alfvénic eigenmodes here but are sometimes also termed global Alfvénic eigenmodes. The branch with $(n=0, m=0)$ corresponds to the GAM. The branch with $(n=0, m=1)$ is referred here as geodesic Alfvénic mode. The gyrokinetic eigenvalue solver LIGKA is used to determine the kinetic continuum branches for $(n=0, m=0)$ and $(n=0, m=1)$. The equations solved by LIGKA are the quasi-neutrality equation $(\mathrm{QN})$ and the gyrokinetic moment equation (GKM) that together with the gyrokinetic equation for the particle distribution functions form a consistent model for electromagnetic perturbations in tokamak geometry $[16,17]$. It can be shown [18] that the implemented equations have the following general dispersion relation as analytical limit [19-21]:

$$
\begin{gathered}
\frac{\omega^{2}}{\omega_{A}^{2}}\left(1-\frac{\omega_{p}^{*}}{\omega}\right)-\bar{k}_{\| m}^{2} R_{0}^{2}=\sum_{a=i, e} 2 \frac{v_{t h, a}^{2}}{\omega_{A}^{2} R_{0}^{2}}\left(-\left[H\left(x_{a, m-1}\right)+H\left(x_{a, m+1}\right)\right]+\right. \\
\left.\tau_{a}\left[\frac{N^{m}\left(x_{a, m-1}\right) N^{m-1}\left(x_{a, m-1}\right)}{D\left(x_{a, m-1}\right)}+\frac{N^{m}\left(x_{a, m+1}\right) N^{m+1}\left(x_{a, m+1}\right)}{D\left(x_{a, m+1}\right)}\right]\right)
\end{gathered}
$$

where $\omega_{A}=v_{A} / R_{0}=\sqrt{B^{2} / \mu_{0} m_{i} n_{i}}$ is the Alfvén frequency and $\bar{k}_{\| m}^{2}$ stands for the parallel wave vector including the toroidal coupling up to first order in $\epsilon$, e.g. $\bar{k}_{\| m}^{2}=\left(k_{\| m}^{2}+k_{\| m+1}^{2} \pm \sqrt{\left(k_{\| m}^{2}-k_{\| m+1}^{2}\right)^{2}+4 \hat{\epsilon}^{2} r^{2} k_{\| m}^{2} k_{\| m+1}^{2}}\right) /\left(2\left(1-\hat{\epsilon}^{2} r^{2}\right)\right)$ where $\hat{\epsilon}=5 r / 2 R_{0}$. Further,

$$
x_{a, m}=\frac{\omega}{\left|k_{\|, m}\right| v_{t h, a}} ; \quad v_{t h, a}=\sqrt{\frac{2 T_{a}}{m_{a}}} ; \quad \omega_{p}^{*}=\sum_{i} \frac{k_{\theta}}{e B n_{i}} \frac{\partial p_{i}}{\partial r} \frac{m_{i} n_{i}}{m_{e f f} n_{e}} \quad \tau=T_{e} / T_{a} .
$$

The definitions for the polynomials $H, N, D$ including the complex plasma dispersion function can be found e.g. in the references $[18,19]$. The upper index $m$ in $N^{m}\left(x_{m-1}\right)$ refers to the poloidal mode number $m$ to be used in $\omega_{m}^{*}=$ $\frac{T_{i}}{e B} k_{m, \theta} \frac{\nabla n}{n}$.

For the geodesic acoustic mode, taking into account the $m= \pm 1$ sidebands, the following dispersion relation can be dervied form the one above for large safety factor $q$ [22, 23]:

$$
\omega_{G}^{2} / \omega_{t, i}^{2}=\hat{\omega}_{G}^{2}=q^{2}\left[\frac{7}{4}+\tau\right]\left[1+\frac{2\left(23+16 \tau+4 \tau^{2}\right)}{q^{2}(7+4 \tau)^{2}}\right]
$$

with $\omega_{t, i}=v_{t h, i} / q R_{0}$. For the $m=1$ branch, the main contribution comes from the $k_{\|}=-1 / q R$ term on the left hand side of equation (3).

The coefficients for the dispersion relation are calculated numerically, based on realistic particle orbits as calculated with the HAGIS code [24]. A Nyquist-contour solver is employed to find the local solutions in the complex plane. Realistic geometry and experimental profiles are used for this analysis. The corresponding spectrum is shown in Fig. 9. At the position where the WCM $(f \sim 130 \mathrm{kHz})$ and the GAM-like mode $(f \sim 8 \mathrm{kHz})$ is observed in the density fluctuations $(\rho \sim 0.98)$ the ( $n=0, m=1$ ) branch is found at $f \sim 140 \mathrm{kHz}$ and the GAM branch $(n=0, m=0)$ is found close to $f \sim 10$ $\mathrm{kHz}$, respectively. Coherent modes are expected slightly below in frequency under the branches since the continuum damping is rather small there.

Motivated by the LIGKA results the mode characteristic has been investigated in detail by calculating the cross-power spectrum of toroidally and poloidally displaced Mirnov coils similar as done in Ref. [25]. The cross-phases $\delta \psi$ and $\delta \theta$ divided by the toroidal $\Delta \psi=0.78$ and poloidal angular distances $\Delta \theta=0.17$, respectively, gives the corresponding mode numbers $n=\delta \psi / \Delta \psi$ and $m=\delta \theta / \Delta \theta$. The cross coherence and mode number are shown in Fig. 10. The WCM can be identified as an $n=0, m=1$ mode. For a coherent mode an increased coherency of the coherent mode in respect to the surrounding frequencies can be expected. This is clearly visible for the toroidal correlation (Fig. 10a). The absence of 
a)

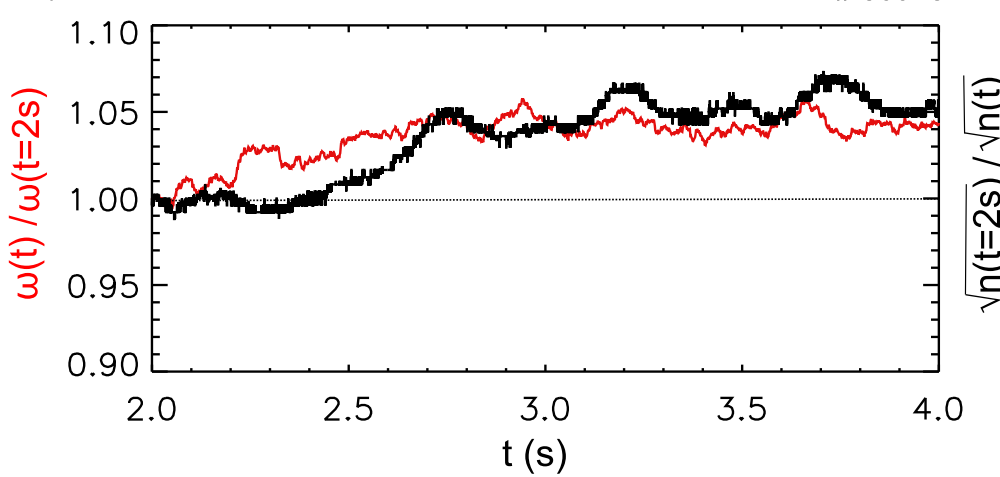

b) \# 30925

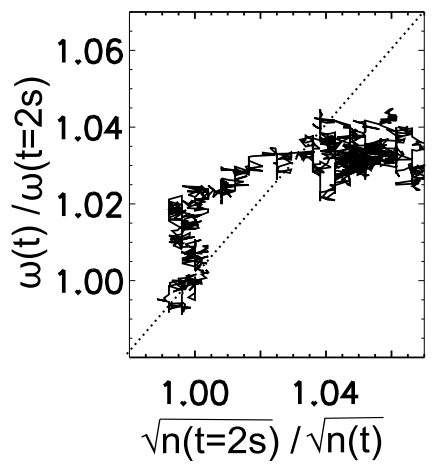

FIG. 8: Frequency of maximum power in the magnetic fluctuations in the range between 100 and $200 \mathrm{kHz}$ and inverse square root of the edge density normalized to their values at the beginning of the I-mode: (a) both against time and (b) against each other.

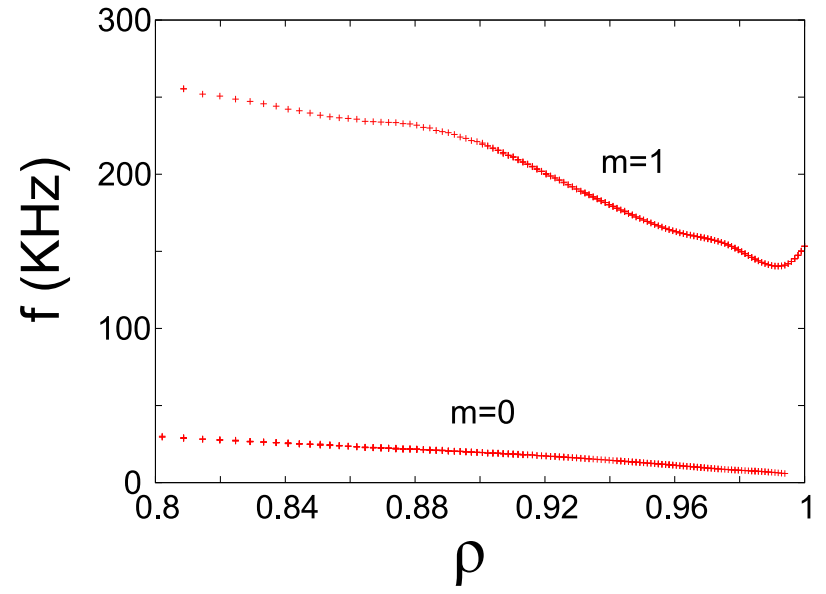

FIG. 9: Spectrum of Alfvénic branch with $n=0$ calculated with LIGKA. The $m=0$ branch corresponds to the geodesic acoustic mode, the $m=1$ branch corresponds to the geodesic Alfvénic mode.

increased coherency in poloidal direction (Fig. 10b) does not support a coherent mode at the WCM frequency in the magnetic fluctuations.

The mode number of the low-frequency mode is more difficult to estimate, its toroidal mode number is between $0 \leq n \leq 1$ the poloidal mode number is around $-1 \leq m \leq 1$. The estimate of the poloidal mode number as done here can be regarded as very rough as the local field line inclination is not taken into account. To obtain a global picture of the modes also the cross-correlation between different poloidally displaced Mirnov coils on a poloidal cross-section has been calculated. As shown in Fig. 11 , the $10 \mathrm{kHz}$ mode exhibits a clear $m=0$ mode structure and the mode close to the WCM-frequency corresponds to a $m=1$.

Whether the observed $m=0$ mode characteristic of the low frequency mode at $10 \mathrm{kHz}$ is characteristic for
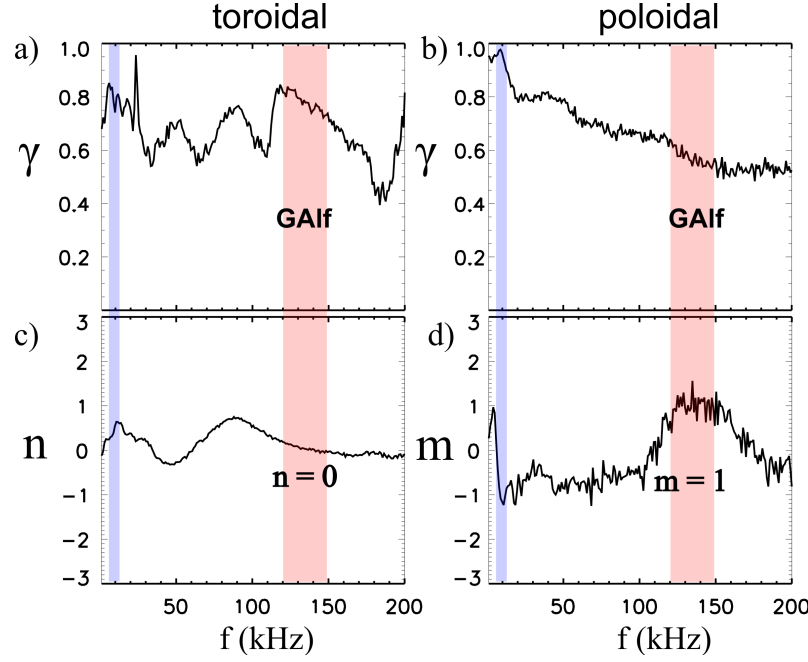

FIG. 10: Cross-coherence (a,b) and toroidal (c) and poloidal mode numbers (d) during I-mode at $4 \mathrm{~s}$ of two mainly toroidally (C04-17 and C05-21) and poloidally (C09-09 and C09-08) displaced Mirnov coils.

a GAM is disputable. The GAM itself is electrostatic. But, it is theoretically expected that the GAM generates a halo of magnetic fluctuations with mode numbers of $m= \pm 2$ just outside the magnetic flux surface of the GAM [26]. This halo has been recently observed in TCV [27]. In this experiment (Fig. 10) the low frequency mode exhibits such a halo, though the poloidal mode numbers are $m= \pm 1$. On the other hand, the observed $(n=0, m=0)$ structure constitutes a zonal magnetic field, which has been predicted to be excited at the GAM frequency for finite $\beta$ turbulence [28]. Here, further systematic studies of the magnetic signature of GAMs in ASDEX Upgrade are needed also in the usual favorable configuration to evaluate if the here observed signature is related to the I-mode configuration in particular. We 

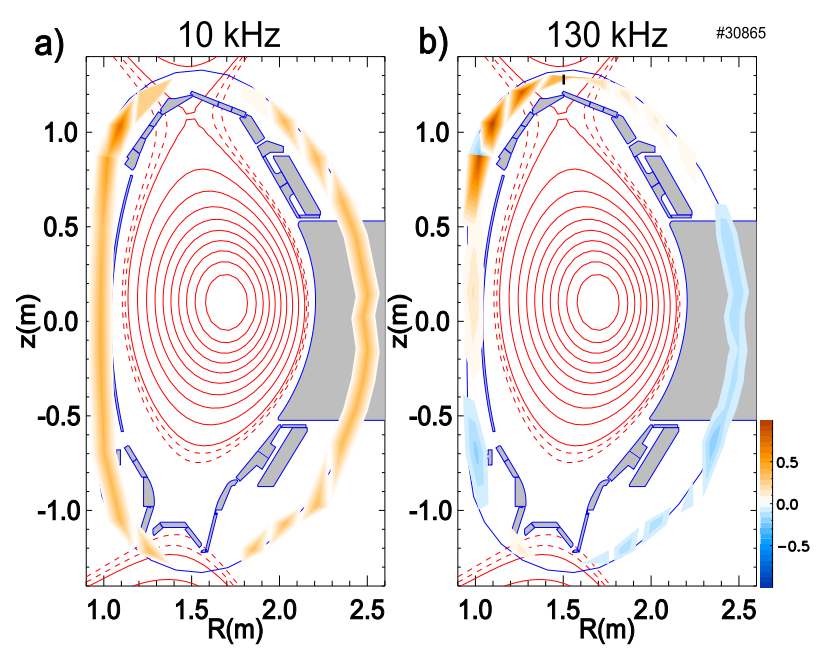

FIG. 11: Poloidal mode structures of the low $(10 \mathrm{kHz})$ and high $(130 \mathrm{kHz})$ frequency oscillations during I-mode at $4 \mathrm{~s}$ shown by the cross-correlation without time lag $(\tau=0)$ of bandpass-filtered signals at the corresponding frequency (10 and $130 \mathrm{kHz}$, respectively) from one Mirnov coil as a reference (cross-correlation is 1) with poloidally displaced Mirnov coils at the same toroidal position.

would expect a Pfirsch-Schlüter-like disturbance of the parallel current and therefore an in-out asymmetry in the fluctuations. The observed perturbation exhibits a strong up-down asymmetry compared to the rather small in-out asymmetry. This is caused in part by the choice of the reference position. In the lower and upper-single null configurations the magnetic configuration exhibits an up-down asymmetry which may lead to an up-down asymmetry in the poloidal magnetic field fluctuations. A detailed study of this asymmetry is left for future studies.

\section{DISCUSSION AND CONCLUSION}

Turbulence during the I-mode has been investigated in ASDEX Upgrade based reflectometry and Mirnov coil measurements. The basic features of the turbulence in the I-mode as observed in Alcator C-Mod are also observed in ASDEX Upgrade:

(i) The characteristic weakly-coherent mode (WCM) is also present in the I-mode in ASDEX-Upgrade. (ii) The WCM is modulated by the GAM found by Doppler reflectometry and envelope analysis of conventional reflectometry as recently observed in Alcator CMod.

In addition (iii) at both frequencies, those of the WCM and the GAM, magnetic fluctuations are present. A local spectral analysis done with the LIGKA code shows that the modes belong to the geodesic Alfvénic branch. The low frequency oscillation in the envelope of the density fluctuations appear at the GAM frequency. The magnetic perturbations at the WCM frequency appear at the geodesic Alfvénic mode frequency. A more detailed analysis of the magnetic fluctuations shows the characteristic toroidal and poloidal mode numbers of the geodesic Alfvénic mode of zero and one, respectively.

The geodesic Alfvénic mode is already present in the L-mode and does not follow variations in frequency of the WCM. Although the WCM and the geodesic Alfvénic mode have to be treated as separated modes their occurrence at a similar frequency let suggest possible interaction or hampering among those modes. A more detailed investigation of the magnetic signatures of the WCM in Alcator C-Mod would be beneficial to proceed in understanding.

The additional occurrence of the geodesic Alfvénic mode in the I-mode in ASDEX Upgrade may reflect some general difference between Alcator C-Mod and ASDEX Upgrade. In the L-mode in most magnetically confined fusion experiments GAMs are regulary observed whereas in Alcator C-Mod up to now only in the I-mode GAMs have been observed [4]. Two conclusions can be deduced, first geodesic effects are stronger in I-mode compared to L-mode, which is also confirmed by the present study, second goedesic effects are rather weak for Alcator CMod compared to other devices. For ASDEX Upgrade the contrary seems likely. Where most devices observe low frequency zonal flows during the transition from $L$ to H-mode [29-35] in ASDEX Upgrade GAMs exhibit this role [36]. As Alcator C-Mod usually operates at higher densities and lower temperature the difference may result from stronger collisional damping. Due to the lower temperature in Alcator C-mod also stronger Landau damping provides a possible explanation. However, Landau damping is unlikely to affect the GAM activity in Alcator C-Mod, as their observations are restricted to the edge region with very high safety factor.
[1] F. Ryter, S. W., B. Brüsehaber, M. Kaufmann, V. Mertens, H. Murmann, A. G. Peeters, J. Stober, J. Schweinzer, H. Zohm, et al., Plasma Phys. \& Controlled Fusion 40, 725 (1998).

[2] D.-G. Whyte, A. E. Hubbard, J. W. Hughes, B. Lipschultz, J. Rice, E. S. Marmar, M. Greenwald, I. Cziegler, A. Dominguez, T. Golfinopoulos, et al., Nucl. Fusion 50, 105005 (2010).
[3] A. E. Hubbard, D. G. Whyte, R. M. Churchill, I. Cziegler, A. Dominguez, T. Golfinopoulos, J. W. Hiughes, J. E. Rice, I. Bespamyatnov, M. J. Greenwald, et al., Phys. Plasmas 18, 331 (2011).

[4] I. Cziegler, P. H. Diamond, N. Fedorczak, P. Manz, G. R. Tynan, M. Xu, A. E. Hubbard, B. Lipschultz, J. M. Sierchio, J. L. Terry, et al., Phys. Plasmas 20, 055904 (2013). [5] A. E. White, P. Phillips, D. G. Whyte, A. E. Hubbard, 
C. Sung, J. W. Hughes, A. Dominguez, J. Terry, and I. Cziegler, Nucl. Fusion 51, 113005 (2011).

[6] U. Stroth, P. Manz, and M. Ramisch, Plasma Phys. \& Controlled Fusion 53, 024006 (2011).

[7] C. Fanack, I. Bouchert, F. Clairet, S. Heuraux, G. Leclert, and X. L. Zou, Plasma Phys. \& Controlled Fusion 38, 1915 (1996).

[8] E. Viezzer, T. Pütterich, G. D. Conway, R. Dux, T. Happel, J. Fuchs, R. M. McDermott, F. Ryter, B. Sieglin, W. Suttrop, et al., Nucl. Fusion 53, 053005 (2013).

[9] Y. Nagashima, K. Hoshino, A. Ejiri, K. Shinohara, Y. Takase, K. Tsuzuki, K. Uehara, H. Kawashima, H. Ogawa, T. Ido, et al., Phys. Rev. Lett. 95, 095002 (2005).

[10] K. J. Zhao, T. Lan, J. Q. Dong, L. W. Yan, W. Y. Hong, C. X. Yu, A. D. Liu, J. Qian, J. Cheng, D. L. Yu, et al., Phys. Rev. Lett. 96, 255004 (2006).

[11] Y. Nagashima, K. Itoh, S.-I. Itoh, A. Fujisawa, M. Yagi, K. Hoshino, S. Shinohara, A. Ejiri, Y. Takase, T. Ido, et al., Plasma Phys. \& Controlled Fusion 49, 1611 (2007).

[12] S. J. Camargo, D. Biskamp, and B. D. Scott, Phys. Plasmas 2, 48 (1995).

[13] P. Manz, M. Xu, S. C. Thakur, and G. R. Tynan, Plasma Phys. \& Controlled Fusion 53, 095001 (2011).

[14] T. Happel, Phys. Rev. Lett. to be submitted (2015).

[15] B. D. Scott, New J. Phys. 7, 92 (2005).

[16] H. Qin, W. M. Tang, and G. Rewoldt, Physics of Plasmas 6, 2544 (1999).

[17] P. Lauber, S. Günter, A. Könies, and S. D. Pinches, Journal Of Computational Physics 226, 447 (2007).

[18] P. Lauber, Physics Reports 533, 33 (2013).

[19] F. Zonca, L. Chen, and R. Santoro, Plasma Physics and Controlled Fusion 38, 2011 (1996).

[20] F. Zonca, L. Chen, A. Botrugno, P. Buratti, A. Cardinali, R. Cesario, and V. Ridolfini, Nuclear Fusion 49, 085009 (2009).

[21] P. Lauber, M. Brüdgam, D. Curran, V. Igochine, K. Sassenberg, S. Günter, M. Maraschek, M. GarciaMunoz, N. Hicks, and the ASDEX Upgrade Team, Plasma Physics and Controlled Fusion 51, 124009 (2009).

[22] H. Sugama and T.-H. Watanabe, Journal of Plasma
Physics 72, 825 (2006), ISSN 1469-7807.

[23] H. Sugama and T.-H. Watanabe, Journal of Plasma Physics 74, 139 (2008).

[24] S. D. Pinches, L. C. Appel, J. Candy, S. E. Sharapov, H. L. Berk, D. Borba, B. N. Breizman, T. C. Hender, K. I. Hopcraft, G. T. A. Huysmans, et al., Computer Physics Communications 111, 133 (1998).

[25] J. Cheng, J. Dong, K. Itoh, L. Yan, W. Hong, K. Zhao, Z. Huang, X. Ji, W. Zhong, D. Yu, et al., Nuclear Fusion 54, 114004 (2014).

[26] C. Wahlberg, Plasma Phys. \& Controlled Fusion 51, 085006 (2009).

[27] C. A. de Meijere, S. Coda, Z. Huang, L. Vermare, T. Vernay, V. Vuille, S. Brunner, J. Dominski, P. Hennequin, A. Krämer-Flecken, et al., Plasma Phys. \& Controlled Fusion 56, 072001 (2014).

[28] N. Chakrabarti, P. N. Guzdar, R. G. Kleva, V. Naulin, J. J. Rasmussen, and P. K. Kaw, Phys. Plasmas 15, 112310 (2008).

[29] T. Estrada et al., Plasma Phys. \& Controlled Fusion 51, 124015 (2009)

[30] L. Schmitz, L. Zeng, T. L. Rhodes, J. C. Hillesheim, E. J. Doyle, R. J. Groebner, W. A. Peebles, K. H. Burrell, and G. Wang, Phys. Rev. Lett. 108, 155002 (2012).

[31] P. Manz, G. S. Xu, B. N. Wan, H. Q. Wang, H. Y. Guo, I. Cziegler, N. Fedorczak, C. Holland, S. H. Müller, S. C. Thakur, et al., Phys. Plasmas 19, 072311 (2012).

[32] G. R. Tynan, M. Xu, P. H. Diamond, J. A. Boedo, I. Cziegler, N. Fedorczak, P. Manz, K. Miki, S. Thakur, L. Schmitz, et al., Nucl. Fusion 53, 073053 (2013).

[33] G. S. Xu, H. Q. Wang, M. Xu, B. N. Wan, H. Y. Guo, P. H. Diamond, G. R. Tynan, R. Chen, N. Yan, D. F. Kong, et al., Nuclear Fusion 54, 103002 (2014).

[34] Z. Yan, G. R. McKee, R. Fonck, P. Gohil, R. J. Groebner, and T. H. Osborne, Phys. Rev. Lett. 112, 125002 (2014).

[35] I. Cziegler, G. R. Tynan, P. H. Diamond, A. E. Hubbard, J. W. Hughes, J. Irby, and J. L. Terry, Plasma Physics and Controlled Fusion 56, 075013 (2014).

[36] G. D. Conway, C. Angioni, F. Ryter, P. Sauter, and J. Vicente, Phys. Rev. Lett. 106, 065001 (2011). 